DOI: $10.21105 /$ joss. 03202

\section{Software}

- Review 七

- Repository こ

- Archive ¿

Editor: Juan Nunez-Iglesias 凹 Reviewers:

- Ca-paxton

- Qabigailmcgovern

Submitted: 10 March 2021 Published: 18 July 2021

\section{License}

Authors of papers retain copyright and release the work under a Creative Commons Attribution 4.0 International License (CC BY 4.0).

\title{
Traja: A Python toolbox for animal trajectory analysis
}

\section{Justin Shenk ${ }^{1,2}$, Wolf Byttner ${ }^{3}$, Saranraj Nambusubramaniyan ${ }^{1}$, and Alexander Zoeller ${ }^{4}$}

1 VisioLab, Berlin, Germany 2 Radboud University, Nijmegen, Netherlands 3 Rapid Health, London, England, United Kingdom 4 Independent researcher

\section{Summary}

There are generally four categories of trajectory data: mobility of people, mobility of transportation vehicles, mobility of animals, and mobility of natural phenomena (Zheng, 2015). Animal tracking is important for fields as diverse as ethology, optimal foraging theory, and neuroscience. Mouse behavior, for example, is a widely studied in biomedical and brain research in models of neurological disease such as stroke. ${ }^{1}$

Several tools exist which allow analyzing mouse locomotion. Tools such as Ethovision (Spink et al., 2001) and DeepLabCut (Mathis et al., 2018) allow converting video data to pose coordinates, which can further be analyzed by other open source tools. DLCAnalyzer ${ }^{2}$ provides a collection of $\mathrm{R}$ scripts for analyzing positional data, in particular visualizing, classifying and plotting movement. B-SOiD (Hsu \& Yttri, 2020) allows unsupervised clustering of behaviors, extracted from the pose coordinate outputs of DeepLabCut. SimBA (sgoldenlab et al., 2021) provides several classifiers and tools for behavioral analysis in video streams in a Windowsbased graphical user interface (GUI) application.

These tools are primarily useful for video data, which is not available for the majority of animal studies. For example, video monitoring of home cage mouse data is impractical today due to housing space constraints. Researchers using Python working with non-visual animal tracking data sources are not able to fully leverage these tools. Thus, a tool that supports modeling in the language of state-of-the-art predictive models (Amirian et al., 2019; Chandra et al., 2019; Liang et al., 2019), and which provides animal researchers with a high-level API for multivariate time series feature extraction, modeling and visualization is needed.

Traja is a Python package for statistical analysis and computational modelling of trajectories. Traja extends the familiar pandas (McKinney, 2010; team, 2020) methods by providing a pandas accessor to the df.traja namespace upon import. The API for Traja was designed to provide an object-oriented and user-friendly interface to common methods in analysis and visualization of animal trajectories. Traja also interfaces well with relevant spatial analysis packages in R (e.g., trajr (McLean \& Volponi, 2018) and adehabitat (Calenge, 2006)), Shapely (Gillies \& others, 2007-), and MovingPandas (Graser, 2019) allowing rapid prototyping and comparison of relevant methods in Python. A comprehensive source of documentation is provided on the home page (http://traja.readthedocs.io).

\section{Statement of Need}

The data used in this project includes animal trajectory data provided by http:/ /www.tecniplast.it, manufacturer of laboratory animal equipment based in Varese, Italy, and Radboud University,

\footnotetext{
${ }^{1}$ The examples in this paper focus on animal motion, however it is useful for other domains.

${ }^{2}$ https://github.com/ETHZ-INS/DLCAnalyzer
} 
Nijmegen, Netherlands. Tecniplast provided the mouse locomotion data collected with their Digital Ventilated Cages (DVC). The extracted coordinates of the mice requires further analysis with external tools. Due to lack of access to equipment, mouse home cage data is rather difficult to collect and analyze, thus few studies have been done on home cage data. Furthermore, researchers who are interested in developing novel algorithms must implement from scratch much of the computational and algorithmic infrastructure for analysis and visualization. By packaging a library that is particularly useful for animal locomotion analysis, future researchers can benefit from access to a high-level interface and clearly documented methods for their work.

Other toolkits for animal behavioral analysis either rely on visual data (Mathis et al., 2018; Sridhar, 2017) to estimate the pose of animals or are limited to the R programming language (McLean \& Volponi, 2018). Prototyping analytical approaches and exploratory data analysis is furthered by access to a wide range of methods which existing libraries do not provide. Python is the de facto language for machine learning and data science programming, thus a toolkit in Python which provides methods for prototyping multivariate time series data analysis and deep neural network modeling is needed.

\section{Overview of the Library}

Traja targets Python because of its popularity with data scientists. The library leverages the powerful pandas library (McKinney, 2010), while adding methods specifically for trajectory analysis. When importing Traja, the Traja namespace registers itself within the pandas dataframe namespace via df .traja.

The software is structured into three parts. These provide functionality to transform, analyse and visualize trajectories. Full details are available at https://traja.readthedocs.io/. The traj ectory module provides analytical and preprocessing functionalities. The models subpackage provides both traditional and neural network-based tools to determine trajectory properties. The plotting module allows visualizing trajectories in various ways.

Data, e.g., $x$ and y coordinates, are stored as one-dimensional labelled arrays as instances of the pandas native Series class. Further, subclassing the pandas DataFrame allows providing an API that mirrors the pandas API which is familiar to most data scientists, thus reducing the barrier for entry while providing methods and properties specific to trajectories for rapid prototyping. Traja depends on Matplotlib (Hunter, 2007) and Seaborn (Waskom, 2021) for plotting and NumPy (Harris et al., 2020) for computation.

\section{Trajectory Data Sources}

Trajectory data as time series can be extracted from a wide range of sources, including video processing tools as described above, GPS sensors for large animals or via home cage floor sensors, as described in the section below. The methods presented here are implemented for orthogonal coordinates $(x, y)$ primarily to track animal centroids, however with some modification they could be extended to work in 3-dimensions and with body part locations as inputs. Traja is thus positioned at the end of the data scientist's chain of tools with the hope of supporting prototyping novel data processing approaches. A sample dataset of jaguar movement (Morato et al., 2018) is provided in the traja.dataset subpackage.

\section{Mouse Locomotion Data}

The data samples presented here ${ }^{3}$ are in 2-dimensional location coordinates, reflecting the mouse home cage $(25 \times 12.5 \mathrm{~cm})$ dimensions. Analytical methods relevant to $2 \mathrm{D}$ rectilinear

\footnotetext{
${ }^{3}$ This dataset has been collected for other studies of our laboratory (Shenk et al., 2020).
} 
analysis of highly constrained spatial coordinates are thus primarily considered.

High volume data like animal trajectories has an increased tendency to have missing data due to data collection issues or noise. Filling in the missing data values, referred to as data imputation, is achieved with a wide variety of statistical or learning-based methods. As previously observed, data science projects typically require at least $95 \%$ of the time to be spent on cleaning, pre-processing and managing the data (Bosch et al., 2021). Therefore, several methods relevant to preprocessing animal data are demonstrated throughout the following sections.

\section{Spatial Trajectory}

A spatial trajectory is a trace generated by a moving object in geographical space. Trajectories are traditionally modelled as a sequence of spatial points like:

$$
T_{k}=\left\{P_{k 1}, P_{k 2}, \ldots\right\}
$$

where $P_{k i}(i \geq 1)$ is a point in the trajectory.

Generating spatial trajectory data via a random walk is possible by sampling from a distribution of angles and step sizes (Kareiva \& Shigesada, 1983; McLean \& Volponi, 2018). A correlated random walk (Figure 1 ) is generated with traja.generate.

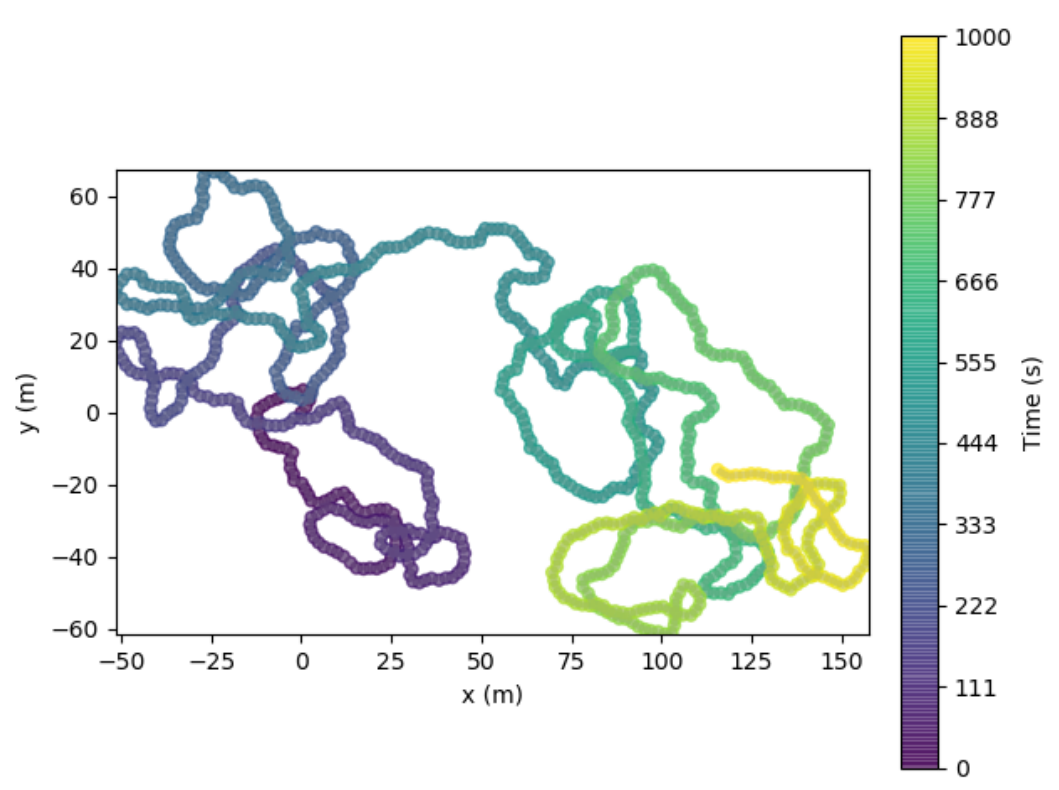

Figure 1: Generation of a random walk

\section{Spatial Transformations}

Transformation of trajectories can be useful for comparing trajectories from various geospatial coordinates, data compression, or simply for visualization purposes.

\section{Feature Scaling}

Feature scaling is common practice for preprocessing data for machine learning (Grus, 2015) and is essential for even application of methods to attributes. For example, a high dimensional 
feature vector $\mathbf{x} \in \mathbb{R}^{n}$ where some attributes are in $(0,100)$ and others are in $(-1,1)$ would lead to biases in the treatment of certain attributes. To limit the dynamic range for multiple data instances simultaneously, scaling is applied to a feature matrix $X=\left\{\mathbf{x}_{\mathbf{1}}, \mathbf{x}_{\mathbf{2}}, \ldots, \mathbf{x}_{\mathbf{N}}\right\} \in$ $\mathbb{R}^{n \times N}$, where $n$ is the number of instances.

Min-Max Scaling To guarantee that the algorithm applies equally to all attributes, the normalized feature matrix $\hat{X}$ is rescaled into range $(0,1)$ such that

$\hat{X}=\frac{X-X_{\min }}{X_{\max }-X_{\min }}$.

Standardization The result of standardization is that the features will be rescaled to have the property of a standard normal distribution with $\mu=0$ and $\sigma=1$ where $\mu$ is the mean (average) of the data and $\sigma$ is the standard deviation from the mean. Standard scores (also known as z-scores are calculated such that

$z=\frac{x-\mu}{\sigma}$.

Scaling Scaling a trajectory is implemented for factor $f$ in scale where $f \in R: f \in$ $(-\infty,+\infty)$.

\title{
Rotation
}

Rotation of a 2D rectilinear trajectory is a coordinate transformation of orthonormal bases $x$ and $y$ at angle $\theta$ (in radians) around the origin defined by

$$
\left[\begin{array}{l}
x^{\prime} \\
y^{\prime}
\end{array}\right]=\left[\begin{array}{ll}
\cos \theta & i \sin \theta \\
\sin \theta & \cos \theta
\end{array}\right]\left[\begin{array}{l}
x \\
y
\end{array}\right]
$$

with angle $\theta$ where $\theta \in R: \theta \in[-180,180]$.

\section{Trip Grid}

One strategy for compressing the representation of trajectories is binning the coordinates to produce an image as shown in Figure 2.

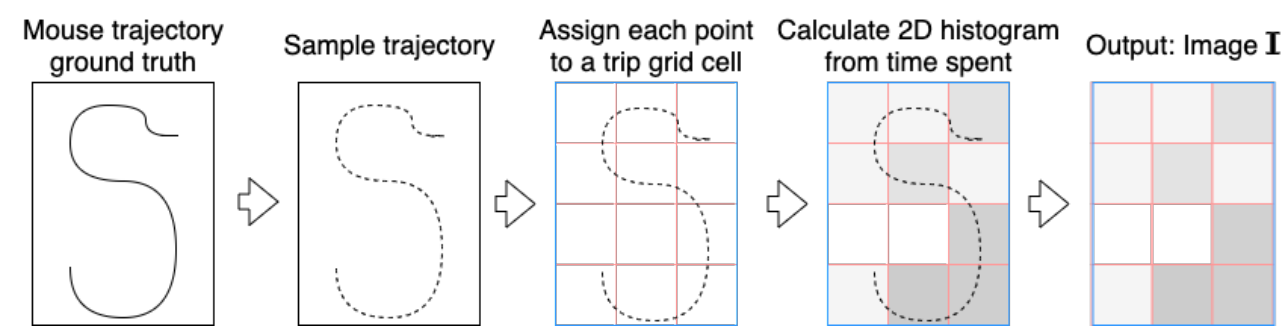

Figure 2: Trip grid image generation from mouse trajectory.

\begin{abstract}
Allowing computation on discrete variables rather than continuous ones has several advantages stemming from the ability to store trajectories in a more memory efficient form. ${ }^{4}$ The advantage is that computation is generally faster, more data can fit in memory in the case of complex models, and item noise can be reduced.

Creation of an $M * N$ grid allows mapping trajectory $T_{k}$ onto uniform grid cells. Generalizing the nomenclature of (Wang, 2017) to rectangular grids, $C_{m n}(1 \leq m \leq M ; 1 \leq n \leq N)$ denotes the cell in row $m$ and column $n$ of the grid. Each point $P_{k i}$ is assigned to a cell

\footnotetext{
${ }^{4}$ In this experiment, for example, data can be reduced from single-precision floating point (32 bits) to 8-bit unsigned integer (uint8) format.
} 
$C(m, n)$. The result is a two-dimensional image $M * N$ image $I_{k}$, where the value of pixel $I_{k}(m, n)(1 \leq m, n \leq M)$ indicates the relative number of points assigned to cell $C_{m n}$. Partionining of spatial position into separate grid cells is often followed by generation of hidden Markov models (Jeung et al., 2007) (see below) or visualization of heat maps (Figure 3).

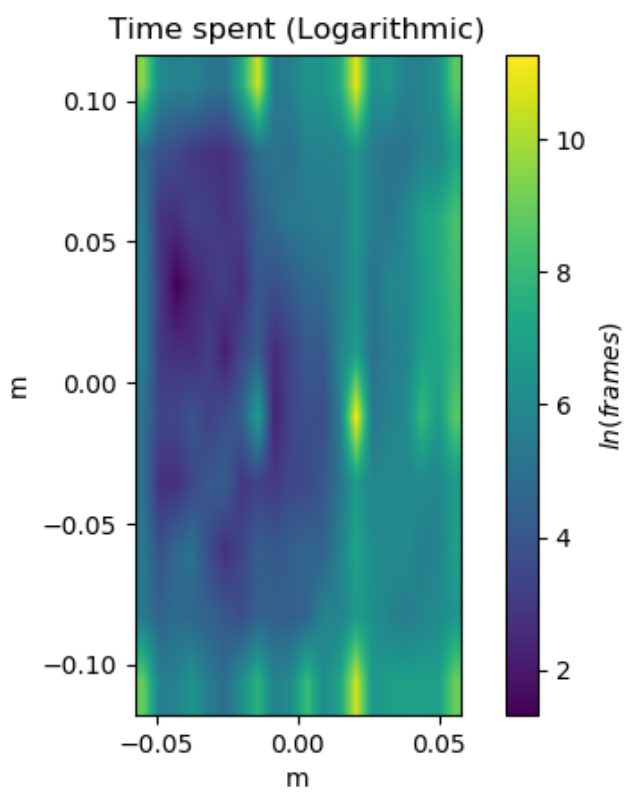

Figure 3: Visualization of heat map from bins generated with df.trip_grid. Note regularly spaced artifacts (bright yellow) in this sample due to a bias in the sensor data interpolation. This type of noise can be minimized by thresholding or using a logarithmic scale, as shown above.

\section{Smoothing}

Smoothing a trajectory can also be achieved with Traja using Savitzky-Golay filtering with smooth_sg (Savitzky \& Golay, 1964).

\section{Resampling and Rediscretizing}

Trajectories can be resampled by time or rediscretized by an arbitrary step length. This can be useful for aligning trajectories from various data sources and sampling rates or reducing the number of data points to improve computational efficiency. Care must be taken to select a time interval which maintains information on the significant behavior. If the minimal time interval observed is selected for the points, calculations will be computationally intractable for some systems. If too large of an interval is selected, we will fail to capture changes relevant to the target behavior in the data.

Resampling by time is performed with resample_time (Figure 4). Rediscretizing by step length is performed with rediscretize. 

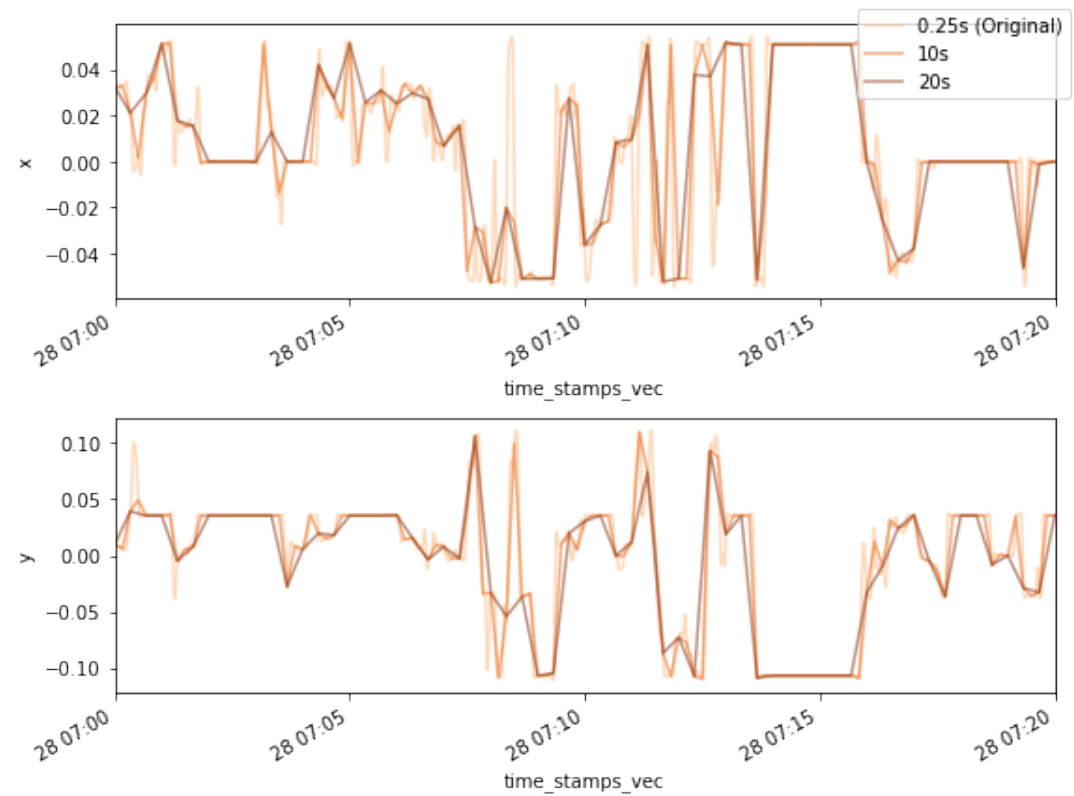

Figure 4: Resampling trajectories by different time scales is performed with resample_time.

For example, the Fortasyn dataset (Shenk et al., 2020) demonstrated in this paper was sampled at $4 \mathrm{~Hz}$ and converted to single-precision floating point data. Pandas dataframes store this data in 4 bytes, thus there are approximately $4.15 \mathrm{MB}^{5}$ bytes required to store data for $\mathrm{x}$ and y dimensions plus an index reference for a single day. In the case of (Shenk et al., 2020), 24 mice were observed over 35 days. This translates to $3.4 \mathrm{~GB}\left(10^{9}\right)$ of storage capacity for the uncompressed datasets prior to feature engineering. Thus resampling can be a useful way to reduce the memory footprint for memory constrained processes that have to fit into a standard laptop with 8 GB memory space. A demonstration of how reduction in precision for trajectory data analysis is provided in Figure 4, as applied to a sample from the Fortasyn experiment (Shenk et al., 2020). Broad effects such as cage crossings, for example, can still be identified while downsampling data to a lower frequency, such as $0.1 \mathrm{~Hz}$, reducing the memory footprint by a factor of $40(4 \mathrm{~Hz} / 0.1 \mathrm{~Hz})$ and providing significant speedups for processing.

\section{Movement Analysis}

Traja includes traditional as well as advanced methods for trajectory analysis.

\section{Distance traveled}

Distance traveled is a common metric in animal studies - it accounts for the total distance covered by the animal within a given time interval. The distance traveled is typically quantified by summing the square straight-line displacement between discretely sampled trajectories (Rowcliffe et al., 2012; Solla et al., 1999). Alternative distance metrics for the case of animal tracking are discussed in (Noonan et al., 2019).

Let $p(t)=\left[p_{x}(t), p_{y}(t)\right]$ be a $2 \times 1$ vector of coordinates on the ground representing the position of the animal at time $t$. Then, the distance traveled within the time interval $t_{1}$ and $t_{2}$ can be computed as a sum of step-wise Euclidean distances

$$
p\left(t_{1}, t_{2}\right)=\Sigma_{t=t_{1}+1}^{t_{2}} d(t),
$$

\footnotetext{
${ }^{5} 4 \times 4 \mathrm{~Hz} \times 60$ seconds $\times 60$ minutes $\times 24$ hours $\times 3$ features $(x, y$, and time $)$
} 
where

$$
d(t)=\sqrt{\left(p_{x}(t)-p_{x}(t-1)\right)^{2}+\left(p_{y}(t)-p_{y}(t-1)\right)^{2}}
$$

is the Euclidean distance between two positions in adjacent time samples.

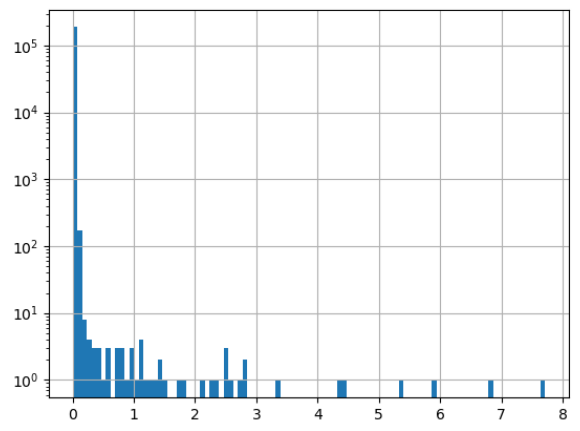

Figure 5: Velocity histogram from one day of mouse activity.

\section{Speed}

Speed or velocity is the first derivative of centroids with respect to time. Peak velocity in a home cage environment is perhaps less interesting than a distribution of velocity observations, as in Figure 5. Additionally, noise can be eliminated from velocity calculations by using a minimal distance moved threshold, as demonstrated in (Shenk et al., 2020). This allows identifying broad-scale behaviors such as cage crossings.

\section{Turn Angles}

Turn angles are the angle between the movement vectors of two consecutive samples. They can be calculated with calc_turn_angles.

\section{Laterality}

Laterality is the preference for left or right turning and a laterality index is defined as:

$$
L I=\frac{R T}{L T+R T}
$$

where RT is the number of right turns observed and LT is the number of left turns observed. Turns are counted within a left turn angle $\in(\theta, 90)$ and right turn angle $\in(-\theta,-90)$. A turn is considered to have a minimal step length.

\section{Periodicity}

Periodic behaviors are a consequence of the circadian rhythm as well as observing expression of underlying cognitive traits. Some basic implementations of periodic analysis of mouse cage data are presented. 


\section{Autocorrelation}

Autocorrelation is the correlation of a signal with a delayed copy of itself as a function of the decay. Basically, it is similarity of observations as a function of the time lag between them. An example is shown in Figure 6.

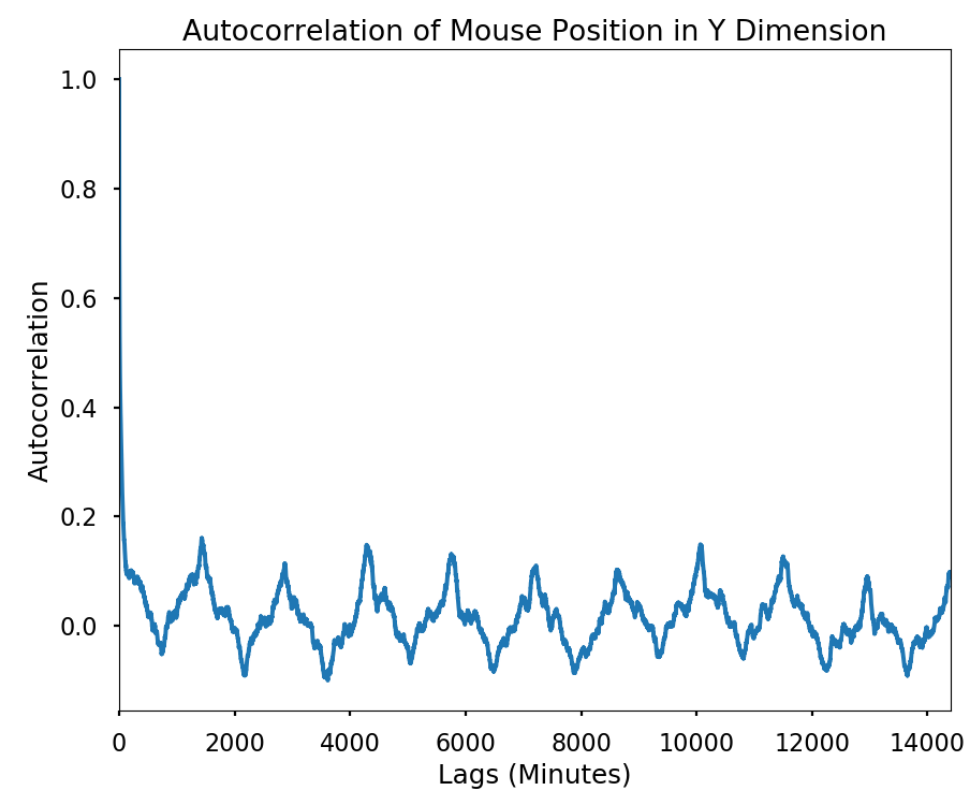

Figure 6: Autocorrelation of the y-dimension reveals daily (1440 minutes) periodic behavior

\section{Power Spectrum}

Power spectrum of a time series signal can be estimated (Figure 7). This is useful for analyzing signals, for example, the influence of neuromotor noise on delays in hand movement (Van Galen et al., 1990). 


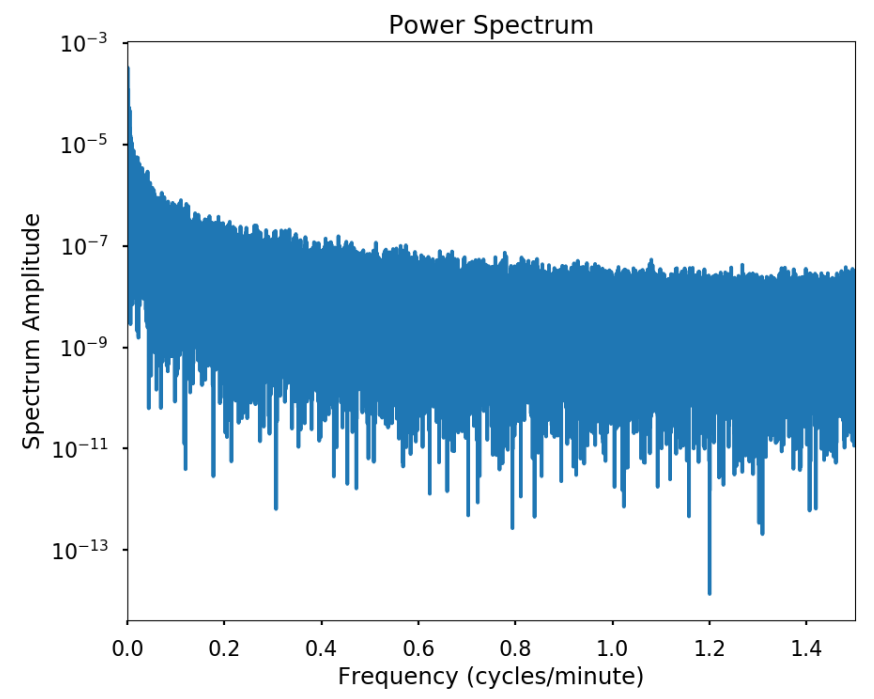

Figure 7: Power Spectral Density. One day of activity reveals fairly smooth power spectral density.

\section{Algorithms and Statistical Models}

\section{Machine Learning for Time Series Data}

Machine learning methods enable researchers to solve tasks computationally without explicit instructions by detecting patterns or relying on inference. Thus they are particularly relevant for data exploration of high volume datasets such as spatial trajectories and other multivariate time series.

\section{Principal Component Analysis}

Principal Component Analysis projects the data into a linear subspace with a minimum loss of information by multiplying the data by the eigenvectors of the covariance matrix. 


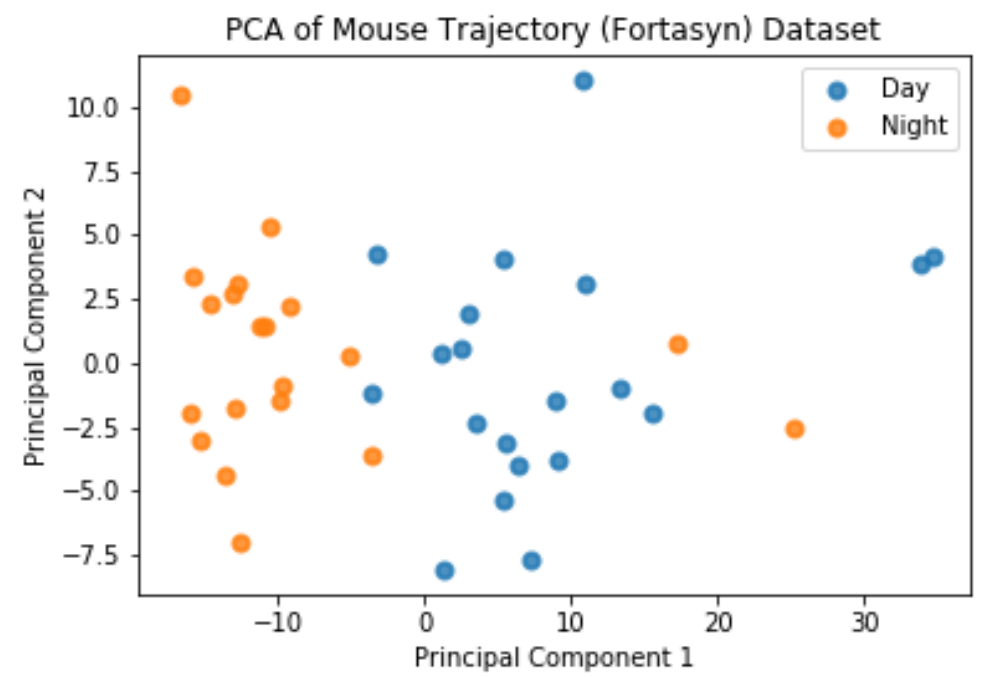

Figure 8: PCA of Fortasyn trajectory data. Daily trajectories (day and night) were binned into $8 \times 8$ grids before applying PCA.

This requires converting the trajectory to a trip grid (see Figure [2(\#fig:tripgridalgo) \{referencetype $=$ "ref" reference="fig:tripgridalgo" $\}]$ ) and performing PCA on the grid in 2D (Figure 8) or 3D (Figure 9). Structure in the data is visible if light and dark time periods are compared.

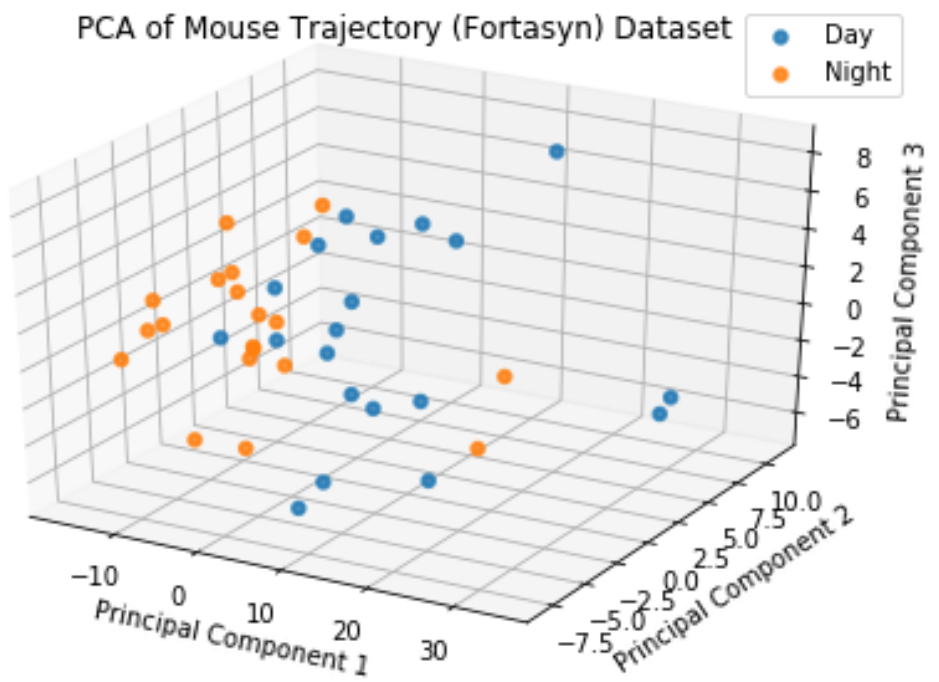

Figure 9: 3D PCA of Fortasyn trajectory data. Daily trajectories (day and night) were binned into $8 \times 8$ grids before applying PCA.

\section{Clustering}

Clustering of trajectories is an extensive topic with applications in geospatial data, vehicle and pedestrian classification, as well as molecular identification. K-means clustering is an iterative unsupervised learning method that assigns a label to data points based on a distance function (Bishop, 2006) (Figure 10). 


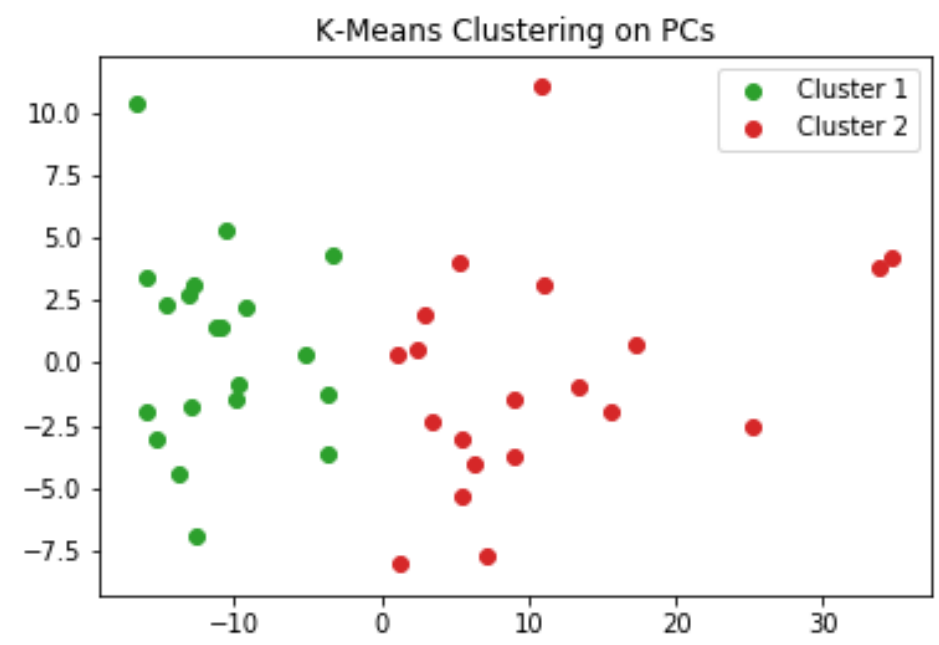

Figure 10: K-means clustering on the results of the PCA shown above reveals a high accuracy of classification, with a few errors. Cluster labels are generated by the model.

\section{Hierarchical Agglomerative Clustering}

Clustering spatial trajectories has broad applications for behavioral research, including unsupervised phenotyping (Huang et al., 2020). For mice, hierarchical agglomerative clustering can also be used to identify similarities between groups, for example periodic activity and location visit frequency (Bak et al., 2009).

\section{Gaussian Processes}

Gaussian Processes is a non-parametric method which can be used to model spatial trajectories. This method is not currently implemented in Traja and is thus outside the scope of the current paper, however the interested reader is directed to the excellent text on Gaussian processes by Rasmussen and Williams (Rasmussen \& Williams, 2006) for a complete reference and (Cox et al., 2012) for an application to spatial trajectories.

\section{Other Methods}

\section{Fractal Methods}

Fractal (i.e. multiscale) methods are useful for analyzing transitions and clustering in trajectories. For example, search trajectories such as eye movement, hand-eye coordination, and foraging can be analyzed by quantifying the spatial distribution or nesting of temporal point processes using spatial Allen Factor analysis (Huette et al., 2013; Kerster et al., 2016).

Recurrence plots and derivative recurrence factor analysis can be applied to trajectories to identify multiscale temporal processes to study transition or nonlinear parameters in a system, such as postural fluctuation (Ross et al., 2016) and synchrony (Shockley et al., 2003) in humans and to movement of animals such as ants (Neves et al., 2017) and bees (Ayers et al., 2015). These methods are not yet implemented in Traja, but are planned for a future release. 


\section{Graph Models}

A graph is a pair $G=(V, E)$ comprising a set of vertices and a set of connecting edges. A probabilistic graphical model of a spatial occupancy grid can be used to identify probabilities of state transitions between nodes. A basic example is given with hidden Markov models below.

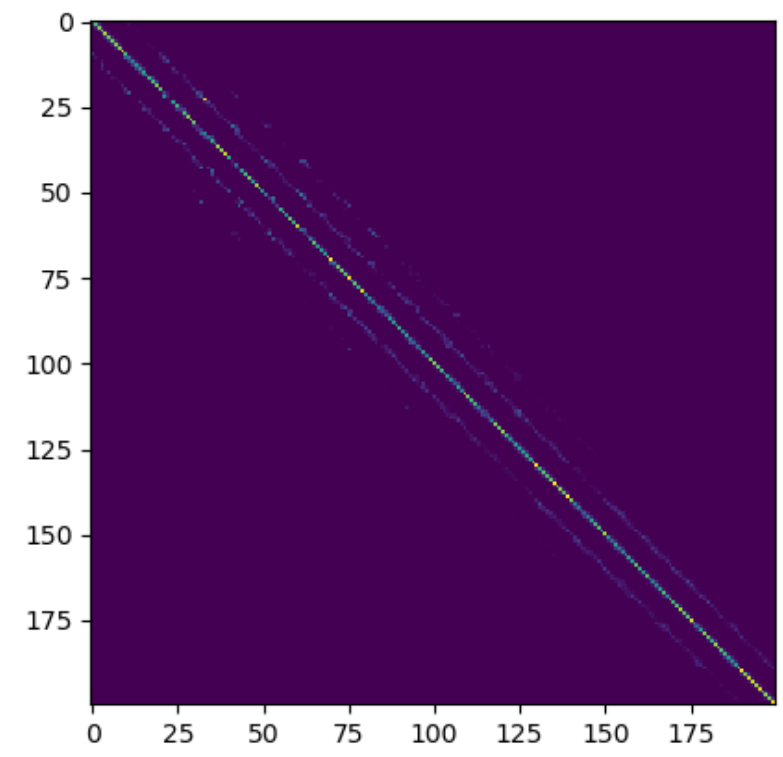

Figure 11: Transition matrix. Rows and columns are flattened histogram of a grid 20 cells high and 10 cells wide. Spatially adjacent grid cells are visible at a spacing of $-11,-10,-9,1,10$, and 11 cells from the diagonal. The intensity of pixels in the diagonal represents relative likelihood to stay in the same position.

\section{Hidden Markov Models}

Transition probabilities are most commonly modelled with Hidden Markov Models (HMM) because of their ability to capture spatial and temporal dependencies. A recent introduction to these methods is available provided by (Patterson et al., 2017). HMMs have successfully been used to analyze movement of caribou (Franke et al., 2004), fruit flies (Holzmann et al., 2006), and tuna (Patterson et al., 2018), among others. Trajectories are typically modelled as bivariate time series consisting of step length and turn angle, regularly spaced in time.

Traja implements the rectangular spatial grid version of HMM with transitions.

The probability of transition from each cell to another cell is stored as a probability within the transition matrix. This can visualized as a heatmap and plotted with plot_transition_ma trix (Figure 11).

\section{Convex Hull}

The convex hull of a subtrajectory is the set $X$ of points in the Euclidean plane that is the smallest convex set to include $X$. For computational efficiency, a geometric k-simplex can be plotted covering the convex hull by converting to a Shapely object and using Shapely's convex_hull method. 


\section{Recurrent Neural Networks}

In recent years, deep learning has transformed the field of machine learning. For example, the current state of the art models for a wide range of tasks, including computer vision, speech to text, and pedestrian trajectory prediction, are achieved with deep neural networks. Neural networks are essentially sequences of matrix operations and elementwise function application based on a collection of computing units known as nodes or neurons. These units perform operations, such as matrix multiplication on input features of a dataset, followed by backpropagation of errors, to identify parameters useful for approximating a function.

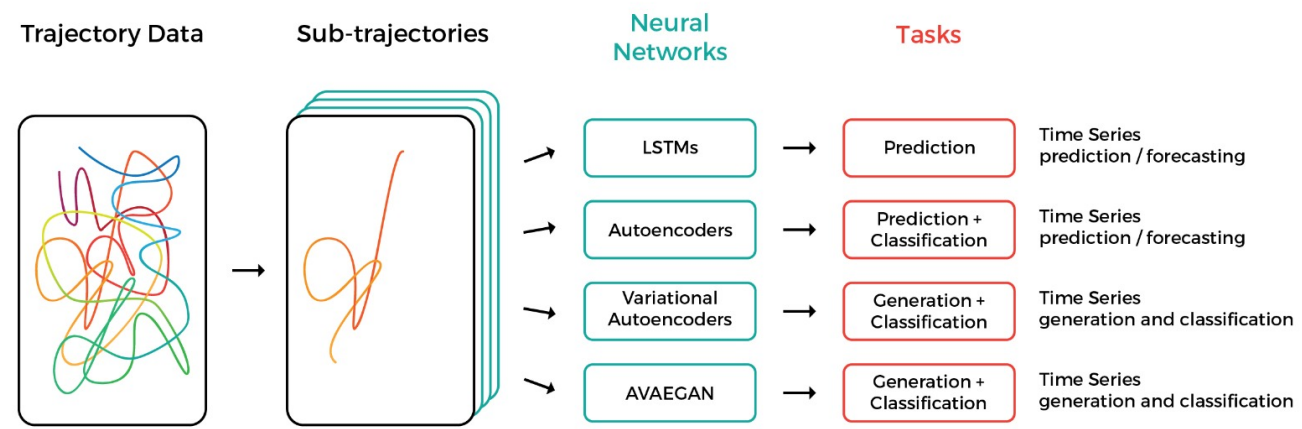

Figure 12: Neural network architectures available in Traja

Recurrent Neural Networks (RNNs) are a special type of Neural Networks that use a state $S\left(t_{i-1}\right)$ from the previous timestep $t_{i-1}$ alongside $\mathrm{X}\left(t_{i}\right)$ as input. They output a prediction $Y\left(t_{i}\right)$ and a new state $S\left(t_{i}\right)$ at every step. Utilising previous states makes RNNs particularly good at analyzing time series like trajectories, since they can process arbitrarily long inputs. They remember information from previous time steps $X\left(t_{i-k}\right), \ldots, X\left(t_{i-1}\right)$ when processing the current time step $X\left(t_{i}\right)$.

Trajectory prediction lets researchers forecast the location and trajectory of animals (Wijeyakulasuriya et al., 2020). Where this technique works well, it is also a sign that the trajectory is highly regular and, fundamentally, follows certain rules and patterns. When tracking an animal live, it would also let researchers predict when it will arrive at a particular location, or where it will go, letting them rig cameras and other equipment ahead of time.

A particularly interesting type of RNN is the Long Short Term Memory (LSTM) architecture. Their layers use stacks of units, each with two hidden variables - one that quickly discards old states and one that slowly does so - to consider relevant information from previous time steps. They can thus look at a trajectory and determine a property of the animal - whether it is sick or injured, say - something that is time-consuming and difficult to do by hand. They can also predict future time steps based on past ones, letting researchers estimate where the animal will go next. LSTMs can also classify trajectories, determining whether a trajectory comes from an animal belonging in a specific category. This lets researchers determine how a controlled or semi-controlled variable (e.g., pregnancy) changes the movement pattern of an animal.

Traja implements neural networks by extending the widely used open source machine learning library PyTorch (Paszke et al., 2019), primarily developed by Facebook Al Research Group. Traja allows framework-agnostic modeling through data loaders designed for time series. In addition, the Traja package comes with several predefined model architectures which can be configured according to the user's requirements. 
Because RNNs work with time series, the trajectories require special handling. The traja.d ataset.MultiModalDataLoader efficiently groups subsequent samples and into series and splits these series into training and test data. It represents a Python iterable over the dataset and extends the PyTorch DataLoader class, with support for

- random, weighted sampling,

- data scaling,

- data shuffling,

- train/validation/test split.

MultiModalDataLoader accepts several important configuration parameters and allows batched sampling of the data. The two constructor arguments $n_{-}$past and $n_{-}$future specify the number of samples that the network will be shown and the number that the network will have to guess, respectively. batch_size is generally in the dozens and is used to regularise the network.

The RNNs also need to be trained - this is done by the high-level Trainer class below. It performs nonlinear optimisation with a Stochastic Gradient Descent-like algorithm. The Trainer class by default implements the Huber loss function (Huber, 1964), also known as smooth $L_{1}$ loss, which is a loss function commonly used in robust regression:

$$
L_{\delta}(a)= \begin{cases}\frac{1}{2} a^{2} & \text { for }|a| \leq \delta, \\ \delta\left(|a|-\frac{1}{2} \delta\right), & \text { otherwise }\end{cases}
$$

In comparison to mean-squared error loss, Huber loss is less sensitive to outliers in data: it is quadratic for small values of $a$, and linear for large values. It extends the PyTorch SmoothL1Loss class, where the $d$ parameter is set to $1 .^{6}$ A common optimization algorithm is ADAM and is Traja's default, but several others are provided as well. Although training with only a CPU is possible, a GPU can provide a $40-100 x$ speedup (Arpteg et al., 2018).

\section{Recurrent Autoencoder Networks}

Traja can also train autoencoders to either predict the future position of a track or classify the track into a number of categories. Autoencoders embed the time series into a time-invariant latent space, allowing representation of each trajectory or sub-trajectory as a vector. A class of well-separated trajectories would then be restricted to a region of the latent space. The technique is similar to Word2vec (Mikolov et al., 2013), where words are converted to a $100+$ dimensional vector. In this approach, forecasting and classification are both preceded by training the data in an autoencoder, which learns an efficient representation of the data for further computation of the target function.

Traja allows training a classifier that works directly on the latent space output - since each class of trajectories converges to a distinct region in the latent space, this technique is often superior to classifying the trajectory itself. Traja trains classifiers for both Autoencoder-style and Variational Autoencoder-style RNNs. When investigating whether animal behavior has changed, or whether two experimental categories of animals behave differently, this unstructured data mining can suggest fruitful avenues for investigation.

\section{References}

Amirian, J., Hayet, J.-B., \& Pettre, J. (2019). Social Ways: Learning Multi-Modal Distributions of Pedestrian Trajectories with GANs. arXiv:1904.09507 [cs]. https://doi.org/10.

\footnotetext{
${ }^{6}$ https://pytorch.org/docs/stable/generated/torch.nn.SmoothL1Loss.html
} 


\section{9/cvprw.2019.00359}

Arpteg, A., Brinne, B., Crnkovic-Friis, L., \& Bosch, J. (2018). Software engineering challenges of deep learning. 2018 44th Euromicro Conference on Software Engineering and Advanced Applications (SEAA), 50-59. https://doi.org/10.1109/seaa.2018.00018

Ayers, C., Armsworth, P., \& Brosi, B. (2015). Determinism as a statistical metric for ecologically important recurrent behaviors with trapline foraging as a case study. Behavioral Ecology and Sociobiology, 69. https://doi.org/10.1007/s00265-015-1948-3

Bak, P., Mansmann, F., Janetzko, H., \& Keim, D. (2009). Spatiotemporal analysis of sensor logs using growth ring maps. IEEE Transactions on Visualization and Computer Graphics, 15, 913-920. https://doi.org/10.1109/TVCG.2009.182

Bishop, C. (2006). Pattern Recognition and Machine Learning. Springer-Verlag. ISBN: 9780-387-31073-2

Bosch, J., Olsson, H. H., \& Crnkovic, I. (2021). Engineering Al Systems: A Research Agenda [Chapter]. In Artificial Intelligence Paradigms for Smart Cyber-Physical Systems. https: //doi.org/10.4018/978-1-7998-5101-1.ch001

Calenge, C. (2006). The package adehabitat for the $r$ software: Tool for the analysis of space and habitat use by animals. Ecological Modelling, 197, 1035.

Chandra, R., Bhattacharya, U., Bera, A., \& Manocha, D. (2019). TraPHic: Trajectory Prediction in Dense and Heterogeneous Traffic Using Weighted Interactions. arXiv:1812.04767 [cs]. https://doi.org/10.1109/cvpr.2019.00868

Cox, G. E., Kachergis, G., \& Shiffrin, R. M. (2012). Gaussian Process Regression for Trajectory Analysis. 6.

Franke, A., Caelli, T., \& Hudson, R. J. (2004). Analysis of movements and behavior of caribou (Rangifer tarandus) using hidden Markov models. Ecological Modelling, 173(2), 259-270. https://doi.org/10.1016/j.ecolmodel.2003.06.004

Gillies, S., \& others. (2007-). Shapely: Manipulation and analysis of geometric objects. toblerity.org. https://github.com/Toblerity/Shapely

Gillies, S., \& others. (2007-). Shapely: Manipulation and analysis of geometric objects. toblerity.org. https://github.com/Toblerity/Shapely

Graser, A. (2019). MovingPandas: Efficient Structures for Movement Data in Python. GI_Forum 2019, Volume 7, 54-68. https://doi.org/10.1553/giscience2019_01_s54

Grus, J. (2015). Data Science from Scratch: First Principles with Python. O'Reilly. ISBN: 978-1-4919-0142-7

Harris, C. R., Millman, K. J., Walt, S. J. van der, Gommers, R., Virtanen, P., Cournapeau, D., Wieser, E., Taylor, J., Berg, S., Smith, N. J., Kern, R., Picus, M., Hoyer, S., Kerkwijk, M. H. van, Brett, M., Haldane, A., R'ı,, J. F. del, Wiebe, M., Peterson, P., ... Oliphant, T. E. (2020). Array programming with NumPy. Nature, 585(7825), 357-362. https: //doi.org/10.1038/s41586-020-2649-2

Holzmann, H., Munk, A., Suster, M., \& Zucchini, W. (2006). Hidden Markov models for circular and linear-circular time series. Environmental and Ecological Statistics, 13(3), 325-347. https://doi.org/10.1007/s10651-006-0015-7

Hsu, A. I., \& Yttri, E. A. (2020). B-SOiD: An open source unsupervised algorithm for discovery of spontaneous behaviors. bioRxiv. https://doi.org/10.1101/770271

Huang, K., Han, Y., Chen, K., Pan, H., Yi, W., Li, X., Liu, S., Wei, P., \& Wang, L. (2020). Mapping Mouse Behavior with an Unsupervised Spatio-temporal Sequence Decomposition Framework. bioRxiv. https://doi.org/10.1101/2020.09.14.295808 
Huber, P. J. (1964). Robust Estimation of a Location Parameter. Annals of Mathematical Statistics, 35(1), 73-101. https://doi.org/10.1214/aoms/1177703732

Huette, S., Kello, C., Rhodes, T., \& Spivey, M. (2013). Drawing from Memory: Hand-Eye Coordination at Multiple Scales. PloS One, 8, e58464. https://doi.org/10.1371/journal. pone.0058464

Hunter, J. D. (2007). Matplotlib: A 2D graphics environment. Computing in Science \& Engineering, 9(3), 90-95. https://doi.org/10.1109/MCSE.2007.55

Jeung, H., Shen, H. T., \& Zhou, X. (2007). Mining Trajectory Patterns Using Hidden Markov Models. In I. Y. Song, J. Eder, \& T. M. Nguyen (Eds.), Data Warehousing and Knowledge Discovery (Vol. 4654, pp. 470-480). Springer Berlin Heidelberg. https://doi.org/10. 1007/978-3-540-74553-2_44

Kareiva, P. M., \& Shigesada, N. (1983). Analyzing insect movement as a correlated random walk. Oecologia, 56(2-3), 234-238. https://doi.org/10.1007/BF00379695

Kerster, B. E., Rhodes, T., \& Kello, C. T. (2016). Spatial memory in foraging games. Cognition, 148, 85-96. https://doi.org/10.1016/j.cognition.2015.12.015

Liang, J., Jiang, L., Niebles, J. C., Hauptmann, A., \& Fei-Fei, L. (2019). Peeking into the Future: Predicting Future Person Activities and Locations in Videos. arXiv:1902.03748 [cs]. https://doi.org/10.1109/cvprw.2019.00358

Mathis, A., Mamidanna, P., Cury, K. M., Abe, T., Murthy, V. N., Mathis, M. W., \& Bethge, M. (2018). DeepLabCut: Markerless pose estimation of user-defined body parts with deep learning. Nature Neuroscience. https://doi.org/10.1038/s41593-018-0209-y

McKinney, Wes. (2010). Data Structures for Statistical Computing in Python. In Stéfan van der Walt \& Jarrod Millman (Eds.), Proceedings of the 9th Python in Science Conference (pp. 56-61). https://doi.org/10.25080/Majora-92bf1922-00a

McLean, D. J., \& Volponi, M. A. S. (2018). Trajr: An R package for characterisation of animal trajectories. Ethology, 124(6), 440-448. https://doi.org/10.1111/eth.12739

Mikolov, T., Sutskever, I., Chen, K., Corrado, G., \& Dean, J. (2013). Distributed representations of words and phrases and their compositionality. http://arxiv.org/abs/1310.4546

Morato, R. G., Thompson, J. J., Paviolo, A., Torre, J. A. de L., Lima, F., McBride, R. T., Paula, R. C., Cullen, L., Silveira, L., Kantek, D. L. Z., Ramalho, E. E., Maranhão, L., Haberfeld, M., Sana, D. A., Medellin, R. A., Carrillo, E., Montalvo, V., MonroyVilchis, O., Cruz, P., ... Ribeiro, M. C. (2018). Jaguar movement database: A GPS-based movement dataset of an apex predator in the Neotropics. Ecology, 99(7), 1691-1691. https://doi.org/10.1002/ecy.2379

Neves, F. M., Viana, R. L., \& Pie, M. R. (2017). Recurrence analysis of ant activity patterns. PLOS ONE, 12(10), 1-15. https://doi.org/10.1371/journal.pone.0185968

Noonan, M. J., Fleming, C. H., Akre, T. S., Drescher-Lehman, J., Gurarie, E., Harrison, A.-L., Kays, R., \& Calabrese, J. M. (2019). Scale-insensitive estimation of speed and distance traveled from animal tracking data. Movement Ecology, 7(1), 35. https://doi.org/10. 1186/s40462-019-0177-1

Paszke, A., Gross, S., Massa, F., Lerer, A., Bradbury, J., Chanan, G., Killeen, T., Lin, Z., Gimelshein, N., Antiga, L., Desmaison, A., Kopf, A., Yang, E., DeVito, Z., Raison, M., Tejani, A., Chilamkurthy, S., Steiner, B., Fang, L., ... Chintala, S. (2019). PyTorch: An imperative style, high-performance deep learning library. In H. Wallach, H. Larochelle, A. Beygelzimer, F. dAlché-Buc, E. Fox, \& R. Garnett (Eds.), Advances in neural information processing systems 32 (pp. 8024-8035). Curran Associates, Inc. http://papers.neurips. cc/paper/9015-pytorch-an-imperative-style-high-performance-deep-learning-library.pdf 
Patterson, T. A., Eveson, J. P., Hartog, J. R., Evans, K., Cooper, S., Lansdell, M., Hobday, A. J., \& Davies, C. R. (2018). Migration dynamics of juvenile southern bluefin tuna. Scientific Reports, 8(1), 1-10. https://doi.org/10.1038/s41598-018-32949-3

Patterson, T. A., Eveson, J. P., Hartog, J. R., Evans, K., Cooper, S., Lansdell, M., Hobday, A. J., \& Davies, C. R. (2018). Migration dynamics of juvenile southern bluefin tuna. Scientific Reports, 8(1), 1-10. https://doi.org/10.1038/s41598-018-32949-3

Patterson, T. A., Parton, A., Langrock, R., Blackwell, P. G., Thomas, L., \& King, R. (2017). Statistical modelling of individual animal movement: An overview of key methods and a discussion of practical challenges. AStA Advances in Statistical Analysis, 101(4), 399-438. https://doi.org/10.1007/s10182-017-0302-7

Patterson, T. A., Parton, A., Langrock, R., Blackwell, P. G., Thomas, L., \& King, R. (2017). Statistical modelling of individual animal movement: An overview of key methods and a discussion of practical challenges. AStA Advances in Statistical Analysis, 101(4), 399-438. https://doi.org/10.1007/s10182-017-0302-7

Rasmussen, CE., \& Williams, CKI. (2006). Gaussian Processes for Machine Learning. Biologische Kybernetik.

Ross, J. M., Warlaumont, A. S., Abney, D. H., Rigoli, L. M., \& Balasubramaniam, R. (2016). Influence of musical groove on postural sway. Journal of Experimental Psychology: Human Perception and Performance, 42(3), 308-319. https://doi.org/10.1037/xhp0000198

Rowcliffe, J. M., Carbone, C., Kays, R., Kranstauber, B., \& Jansen, P. A. (2012). Bias in estimating animal travel distance: The effect of sampling frequency. Methods in Ecology and Evolution, 3(4), 653-662. https://doi.org/10.1111/j.2041-210X.2012.00197.x

Savitzky, Abraham., \& Golay, M. J. E. (1964). Smoothing and Differentiation of Data by Simplified Least Squares Procedures. Analytical Chemistry, 36(8), 1627-1639. https: //doi.org/10.1021/ac60214a047

sgoldenlab, Choong, J. J., Nilsson, S., Islam, A., \& sophihwang26. (2021). Sgoldenlab/simba: SimBA: Release v1.3 (Version v1.3) [Computer software]. Zenodo. https://doi.org/10. $5281 /$ zenodo. 4521178

Shenk, J., Lohkamp, K. J., Wiesmann, M., \& Kiliaan, A. J. (2020). Automated Analysis of Stroke Mouse Trajectory Data With Traja. Frontiers in Neuroscience, 14. https://doi. org/10.3389/fnins.2020.00518

Shockley, K., Santana, M.-V., \& Fowler, C. (2003). Mutual interpersonal postural constraints are involved in cooperative conversation. Journal of Experimental Psychology. Human Perception and Performance, 29, 326-332. https://doi.org/10.1037/0096-1523.29.2.326

Solla, S. R. D., Bonduriansky, R., \& Brooks, R. J. (1999). Eliminating autocorrelation reduces biological relevance of home range estimates. Journal of Animal Ecology, 68(2), 221-234. https://doi.org/10.1046/j.1365-2656.1999.00279.x

Spink, A. J., Tegelenbosch, R. A. J., Buma, M. O. S., \& Noldus, L. P. J. J. (2001). The EthoVision video tracking system-A tool for behavioral phenotyping of transgenic mice. Physiology \& Behavior, 73(5), 731-744. https://doi.org/10.1016/S0031-9384(01)00530-3

Sridhar, V. H. (2017). Vivekhsridhar/tracktor: tracktor (tracktor) [Computer software]. Zenodo. https://doi.org/10.5281/zenodo.1134016

team, T. pandas development. (2020). Pandas-dev/pandas: pandas (latest) [Computer software]. Zenodo. https://doi.org/10.5281/zenodo.3509134

Van Galen, G. P., Van Doorn, R. R., \& Schomaker, L. R. (1990). Effects of motor programming on the power spectral density function of finger and wrist movements. Journal of Experimental Psychology: Human Perception and Performance, 16(4), 755-765. https://doi.org/10.1037/0096-1523.16.4.755 
Wang, X. (2017). Modeling Trajectory as Image: Convolutional Neural Networks for Multi-scale Taxi Trajectory Prediction. https://www.academia.edu/34767293/ Modeling_Trajectory_as_Image_Convolutional_Neural_Networks_for_Multi-scale_ Taxi_Trajectory_Prediction

Waskom, M. L. (2021). Seaborn: Statistical data visualization. Journal of Open Source Software, 6(60), 3021. https://doi.org/10.21105/joss.03021

Wijeyakulasuriya, D. A., Eisenhauer, E. W., Shaby, B. A., \& Hanks, E. M. (2020). Machine learning for modeling animal movement. PLOS ONE, 15(7), 1-30. https://doi.org/10. 1371/journal. pone. 0235750

Zheng, Y. (2015). Trajectory Data Mining: An Overview. ACM Transaction on Intelligent Systems and Technology. https://www. microsoft.com/en-us/research/publication/ trajectory-data-mining-an-overview/ 